\title{
Income Inequality in a Job-Search Model With Heterogeneous Discount Factors
}

\author{
Rubens Penha Cysne \\ Professor at the Graduate School of Economics of the Getulio Vargas Foundation
}

\begin{abstract}
This paper investigates the income inequality generated by a job-search process when different cohorts of homogeneous workers are allowed to have different degrees of impatience. Using the fact the average wage under the invariant Markovian distribution is a decreasing function of the discount factor (Cysne 2004, 2006), I show that the Lorenz curve and the between-cohort Gini coefficient of income inequality can be easily derived in this case. An example with arbitrary measures regarding the wage offers and the distribution of time preferences among cohorts provides some insights into how much income inequality can be generated, and into how it varies as a function of the probability of unemployment and of the probability that the worker does not find a job offer each period.
\end{abstract}

Keywords: Income Inequality, Job Search, Impatience, Time Preference

JEL Classification: D63, J64

\section{Resumo}

Este trabalho investiga a desigualdade de renda salarial gerada por um modelo usual de procura de trabalho (job search), quando se admite que diferentes coortes (cohorts) de trabalhadores têm diferentes graus de impaciência (desconto intertemporal). Usando fortemente o fato (provado em Cysne $(2004,2006)$ ) de que o salário médio em cada coorte é função crescente do fator de desconto intertemporal, o trabalho mostra que o coeficiente de Gini pode ser facilmente calculado, e como calculá-lo. Usando uma distribuição Beta para o coeficiente de desconto entre coortes, o trabalho provê um exemplo que dá uma medida quantitativa de quanto se pode gerar em termos de desigualdade usando a heterogeneidade de fatores de desconto.

\footnotetext{
* Received in October 2005, accepted in January 2006. I am thankful to participants of workshops at the Graduate School of Economics of the Getulio Vargas Foundation and at the Department of Economics of the University of Chicago, for their comments.

Email address: rubens@fgv.br.
}

Revista EconomiA 


\section{Introduction}

A condition for a stochastic dynamic model to be of some use in the understanding of income distribution is that it delivers an endogenous distribution of incomes. Within this class of models, job-search models are certainly among the simplest. In this case, the endogenous distribution is given by the invariant distribution of wages in the economy generated by the rules defining the job search. A nice feature of studying income inequality in this category of models is that, as we shall see, the Gini coefficient of income inequality can be parameterized in terms of some specific characteristics of the job market, such as the probability of unemployment and the probability that an unemployed worker finds no job offer in a certain period.

Pissarides (1974) uses a job-search model to compare the income distribution ${ }^{1}$ of two cohorts ${ }^{2}$ with different degrees of risk aversion. This author argues that cohorts with higher risk aversion will have a lower reservation wage and, because the wages of employed workers will have a greater range, the income distribution will be worse.

Pissarides does not explicit derive the stationary distribution generated by the interactions of the transition functions implicit in his model. Moreover, his work concentrates only on comparing (between two cohorts) within-cohort inequalities, not formally deriving a measure of inequality among cohorts.

In this work I complement Pissaride's analysis by pursuing answers to three different questions. First, instead of dealing with heterogenous degrees of risk aversion, I allow consumers in different cohorts to have different discount-factor parameters. Second, in each cohort I concentrate the analysis on the invariant Markovian distribution of all wages that a representative has along his (supposedly infinite) life time. Third, I analyze inequality among cohorts, rather than within cohorts.

The inequality among cohorts is generated by the fact that more patient workers have higher reservation wages and higher average wages as well (see Cysne (2004, 2006) for a demonstration of this fact).

The paper proceeds as follows. Section 2 is used for the presentation of the general model, fully characterizing the job-search problem within a cohort. Section 3 is dedicated to the derivation of the invariant-distribution between-cohort Gini coefficient of income inequality. Section 4 is used to make quantitative assessments of the model, based on an example with arbitrary measures of the wage offers within cohorts and of the distribution of discount factors among cohorts. Section 5 concludes.

\footnotetext{
1 More rigorously, we are dealing here with wage inequality. However, transfers and capital income usually represent only a small fraction of most households' total income. For the United States, for instance, following the 1992 SCF (Survey of Consumer Finances), transfers and capital income account in average for only around $28 \%$ of the total income of the households surveyed. This percentage tends to be even lower in developing countries.

2 In this work, a cohort is defined as a group of homogenous workers/consumers.
} 


\section{The Model}

The basic model presented here is the same as the one presented in Cysne (2004, 2006), which in turn draws on Stokey and Lucas's (1989) version of McCall's (1970) model. The givens of the model, a partial-equilibrium one, ${ }^{3}$ are the distribution of wage offers (the same for all workers and along all cohorts), the distribution of the discount-factor parameter among cohorts of workers, the probability of layoffs and the probability that, each period, the worker does not get any job offer next period.

In the measurable space $\left([0,1), \mathcal{B}_{[0,1)}, \mathcal{L}\right), \mathcal{B}_{[0,1)}$, standing for the borelians in $[0,1)$ and $\mathcal{L}$ for the Lebesgue measure, consider a continuum of cohorts, indexed by $j$, each cohort with a large number of homogenous workers. Cohorts differ only in terms of their discount-factor parameter. The distribution of discount factors $\beta_{j} \in[0,1)$ along these cohorts is determined by:

$$
\beta_{j}=H^{-1}(j), H^{\prime}(.)>0
$$

In equation (1), $j$ has a uniform distribution in $[0,1)$ and $H$ stands for a cumulative probability distribution of a random variable taking values in $[0,1)$. The isomorphism (1) allows us to put different probability measures " $m$ " in the space where the discount-factor parameters take value $\left(\right.$ also $\left.\left([0,1), \mathcal{B}_{[0,1)}\right)\right)$. For instance, if $H$ is the cumulative distribution function of a Beta $(s, v)$ random variable, then the $\beta_{j}^{\prime} s$ will be distributed as a Beta $(s, v)$. Note that having $H^{\prime}()>$.0 allows us to identify each cohort $j$ with its discount-factor parameter $\beta_{j}$.

For $0<D<\infty$, consider also the second measurable space $\left(\Omega, \mathcal{B}_{[0, D]}, p\right), \Omega$ a generic sample space and, in this measurable space, the measure $q$ induced by the wage-offers function $w: \Omega \rightarrow[0, D]$. In the induced space $\left([0, D], \mathcal{B}_{[0, D]}, q\right)$, denote by $F(t)$ the distribution function that $(q-a . e$. -uniquely) determines the measure $q: F(t)=p(w \leq t)$.

By assumption, in each cohort, there are two states regarding the consumer's optimization problem: $w$ and 0 . State " $w$ " corresponds to a job offer of $w$ at hand, and state " 0 " to no job offer. In state $w$ the worker can accept or turn down the offer. If he accepts it, by assumption he stays employed with that wage till he is laid off (which happens with probability $\theta$ ). If he does not accept the offer or if he gets no offer, he remains in state 0 . Being in state zero the only thing he can do is wait again for a job offer next period, which happens with probability $(1-\alpha)$. The individual is not allowed to search while in his job. The job offers are drawn from $[0, D]$ according to the measure $q . q$ is known by all workers in all cohorts. Workers are not allowed to borrow or to lend. Their consumption $c_{t}$ is equal to their income $w_{t}$ in each period. Consumers in cohort $j$ maximize the expected present value of

\footnotetext{
3 The reader interested in a job-search model with a more general-equilibrium flavour, including endogenous creation and destruction of jobs, can refer to Mortensen and Pissarides (1994).
} 
their consumption $E\left(\sum_{t=0}^{\infty} \beta_{j}^{t} c_{t}\right)$. From now on I will only use the index $j$ when necessary.

With $v(w)$ stating for the value function, $\bar{w}$, the reservation wage is determined by Cysne $(2004,2006)$ :

$$
\bar{w}=\frac{\beta(1-\alpha)}{1-\beta(1-\theta)} \int_{[\bar{w}, D]}\left(w^{\prime}-\bar{w}\right) d F\left(w^{\prime}\right)
$$

Following the analysis in Stokey et al. (1989), c. 10, which displays the particular case in which $\alpha=0$, and equivalently to Cysne $(2004,2006)$, the reservation wage $\bar{w}(j)$ divides $[0, D]$ into two regions: the acceptance region $A(\beta)=[\bar{w}(\beta), D]$ and the non-acceptance region $A^{c}(\beta)=[0, \bar{w}(\beta)]$. Consider a new measure $\lambda_{t}$ in $\left([0, D], \mathcal{B}_{[0, D]}\right)$, representing the effective wage of workers of a certain cohort $j$ (the $j$ is omitted), in period $t$. Note that $A$ and $A^{c}$ depend on the value of $\beta$.

The rules of the optimization followed by the worker define a transition function $P:[0, D] \times \mathcal{B}_{[0, D]} \rightarrow[0,1]$ of wage offers. If the current state (given by the wage offer) is $w \in A^{c}$, the probability of having an offer next period in any borelian $B \subset[0, D]$ is given by $(1-\alpha) q(B)+\alpha$, if $0 \in B$, and $(1-\alpha) q(B)$ if $0 \notin B$. Alternatively, if the current state is $w \in A$, the worker can only lose his job (with probability $\theta$ ) or keep the same wage next period. Therefore, with probability zero he will have a wage next period in a borelian $B$ that does not contain either 0 or $w$. If the borelian $B$ contains 0 , but not $w$, or $w$ but not zero, the transition probabilities are, respectively, $\theta$ and $1-\theta$. If it contains both, since these are disjoint events (because $0 \notin A), P(w, B)=1$.

A worker will be offered a wage in the acceptance region $A$ in period $t+1$ if either she already had this wage in $A$ in period $t$ and is not laid off or if she was unemployed, receives a wage offer, and this wage offer falls in $A$. Formally:

$$
\lambda_{t+1}(A)=\lambda_{t}(A)(1-\theta)+\lambda_{t}\left(A^{c}\right)(1-\alpha) q(A)
$$

Taking limits, ${ }^{4}$ this leads to the following invariant measure of the acceptance region $\mathrm{A}\left(\lambda=\lim _{t \rightarrow \infty} \lambda_{t}\right)$ :

$$
\lambda(A)=\frac{(1-\alpha) q(A)}{\theta+(1-\alpha) q(A)}
$$

Since $A \cup A^{c}=[0, D]$ and $A \cap A^{c}=\phi$ :

$$
\lambda\left(A^{c}\right)=1-\lambda(A)=\frac{\theta}{\theta+(1-\alpha) q(A)}
$$

\footnotetext{
4 It is easy to show that the adjoint operator (defined in the linear space of signed measures endowed with the total variation norm) defined by the transition function above has one and only one unique fixed point. This fact allows us to take limits in (3). This fixed point corresponds to the invariant measure of wage offers $\lambda$, defined in $\left([0, D], \mathcal{B}_{[0, D]}\right)$, which is the invariant measure we are looking for.
} 
A worker will have a wage offer in a set $C \subset A$ in data $t+1$ if either she already has a wage $w \in C$ and keeps it, or if she is unemployed, happens to receive a wage offer, and this wage offer is in $C$. Formally, for $C \subset A$ :

$$
\lambda_{t+1}(C)=\lambda_{t}(C)(1-\theta)+\lambda_{t}\left(A^{c}\right)(1-\alpha) q(C)
$$

Using (4) and (5), the invariant measure of wage is now given by:

$$
\lambda(C)=\frac{(1-\alpha) q(C)}{\theta+(1-\alpha) q(A)}
$$

The observed stationary distribution of wages in this model, $\lambda^{*}$, is a mixed distribution in $[0, D]$. For,$S \in \mathcal{B}_{[0, D]}$ :

$$
\lambda^{*}(S)= \begin{cases}\frac{\theta}{\theta+(1-\alpha) q(A)}, & \text { if } S=\{0\} \\ 0, & \text { if } S \subset[0, \bar{w}] \\ \frac{(1-\alpha) q(S)}{\theta+(1-\alpha) q(A)}, & \text { if } S \subset A\end{cases}
$$

The average wage of all workers (including employed and unemployed workers) in each cohort $j$ is then given by:

$$
w_{A}(j)=\int_{[\bar{w}(j), D]} \frac{(1-\alpha) w d F(w)}{\theta+(1-\alpha) q(A(j))}
$$

where $\bar{w}(j)$ follows $(2)$. As shown in Cysne $(2004,2006), w_{A}$ is an increasing function of the discount-factor parameter and, given (1), of $j$ as well.

\section{Income Inequality}

The existence of different discount factors between cohorts leads to a between-cohort income inequality. The Proposition below provides an expression for the Gini coefficient as a function of the exogenous measure to be put into the discount-factor parameters. Take $a \in[0,1)$.

For use in Proposition 1, make equal to $\bar{w}_{A}$ the average of the average wage in each cohort (given by (8)) considering the respective distribution of time preferences among cohorts:

$$
\bar{w}_{A}=\int_{0}^{1} w_{A}(u) d m(u)
$$


Proposition 1. The between-cohort Gini coefficient of income inequality associated with the problem outlined above is given by: ${ }^{5}$

$$
G=1-\frac{2}{\bar{w}_{A}} \int_{[0,1)}\left[\int_{\left[0, \beta_{j}\right)} w_{A}(u) d m(u)\right] d m\left(\beta_{j}\right)
$$

Proof. The Lorenz curve $L(j)$ plots the percentage of total income earned by the economic agents of a certain cohort, when these cohorts are ordered from those with lower average income to those with higher average income. As shown by Cysne $(2004,2006)$, there is an isomorphism linking the average wage $w_{A}$ to the discount-factor parameter $\beta$ (because $w_{A}^{\prime}(\beta)>0$ ). This fact implies that, by ordering the population by $\beta_{j}$ we are, automatically, also ordering it by income, as required in the construction of the Lorenz curve. Therefore, in this case the Lorenz curve can be easily written as:

$$
L(j)=\frac{1}{\bar{w}_{A}} \int_{\left[0, \beta_{j}\right)} w_{A}(u) d m(u)
$$

The Gini coefficient by definition is equal to $1-2 *$ (area under the Lorenz curve), the abcissa runing from 0 to 1 . Therefore, taking into consideration the measure $m$ :

$$
G=1-2 \int_{[0,1)} L(u) d m(u)
$$

Note that above we are using the fact that the measure of cohorts with discount factor equal or less than a certain constant $a \in[0,1)$ is given by: $P\left(\beta_{j} \leq a\right)=$ $P\left(H^{-1}(j) \leq a\right)=P(j \leq H(a))=H(a)=m([0, a])$. This is also equivalent to the measure of people with income less or equal than $w_{A}\left(\beta_{j}\right)$.

(10) follows trivially from (11) and (12).

\section{Quantitative Assessments}

This is as far as one can go without specifying the measures $q$ (of wage offers) and $m$ (of the distribution of discount factors among cohorts ). In order to get some idea of the numbers generated by the analysis developed here, from now on I will assume that measure $q$ is the Lebesgue measure in $[0,1]$ (this measure allows for closed-form solutions to the reservation wage, the average wage, and the Gini coefficient), and that $m$ has a density with respect to the Lebesgue measure given by the density of a Beta $(114.5,1.01)$ distribution. The parameters of this distribution have been chosen in order to make the (monthly) average equal to $114.5 /(114.5+1.01)=0.9913$, which corresponds to an yearly average discount factor of 0.900. Some numerical results are presented in the example below.

5 When a density $\phi(u)$ of measure $m$ with respect to the Lebesgue measure in $\mathbb{R}$ is defined, $\int_{0}^{\beta_{j}} w_{A}(u) d m(u)$ can be more familiarly written as $\int_{0}^{\beta_{j}} w_{A}(u) \phi(u) d u$. 
Example 1 This example assumes that in each period the workers face a probability $\theta$ of layoff, a probability $\alpha$ of not finding a wage offer and measures $q$ and $m$, specified as above. Using (2), (8), (9) and (10), the Gini coefficient reads:

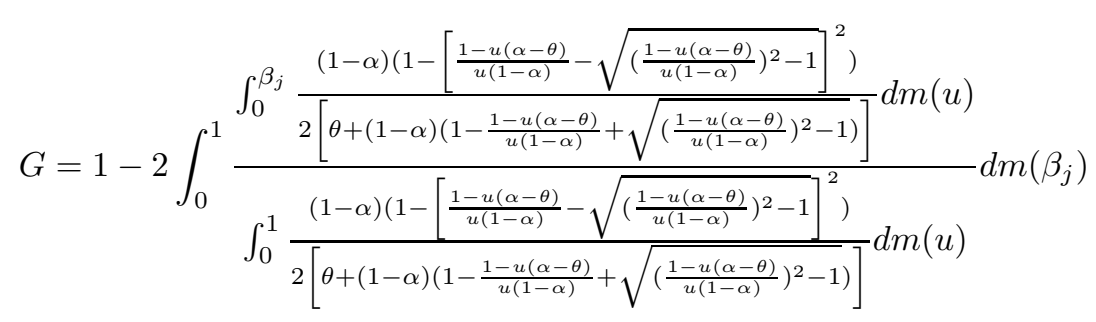

The values of the Gini coefficients, for different values of $\theta$ and $\alpha$, are shown in Table 1 below: 6

\section{Table 1}

Gini Coefficient

$$
\text { Theta }
$$

0

Alpha $0 \quad 0.1207 \quad 0.1049$

$\begin{array}{lll}0.2 & 0.1224 & 0.1049\end{array}$

Note in Table 1 that the between-cohort inequality decreases when the probability of layoff increases. An increase in $\theta$ has two effects. First, it decreases the reservation wages of all cohorts, thereby making it more likely that low wage offers are taken by workers in some cohorts. Second, having workers unemployed more frequently, the average wages in all cohorts decrease, impoverishing all cohorts at the same time, and decreasing inequality.

The sensibility of the income distribution to the probability of not finding a job offer each period, though, in the range of values of theta and alpha in which we derived the results of Table 1, was of a different sign and very small.

\section{Conclusions}

In this paper I have investigated income inequality among cohorts of homogenous workers, when each cohort is characterized by a different degree of impatience. The work can be regarded as complementary to Pissarides's (1974), who used a job-search model to study within-cohort inequalities.

\footnotetext{
6 The absolute value of the numbers in Table 1, of course, vary according to how fine the numerical integration is carried out. The qualitative dependence of the Gini coefficient on the parameter values alpha and tetha, though, is not subject to this problem.
} 
Using the result obtained by Cysne $(2004,2006)$, that the average wage in each cohort based on the invariant Markov measure is a decreasing function of impatience, I have shown how to derive the Lorenz curve and the between-cohort Gini coefficient of income inequality. Next, I have provided an example, based on arbitrary measures concerning the job offers and the distribution of discount factors among the different cohorts. The example helps the to understand how the parameter values affect the Gini coefficient, and gives an idea of the amount of inequality that can be generated on account of discrepancies in discount factors among groups of workers.

The numbers obtained indicate that the degree of inequality is decreasing in the probability of unemployment and nondecreasing in the probability that unemployed workers in each cohort do not find job offers each period. The probability of unemployment has shown to have a greater impact over the generation of income inequality than the probability of finding job offers.

\section{References}

Cysne, R. P. (2004). A search-theoretic explanation for the negative correlation between labor income and impatience. Ensaio Econômico 558, EPGE/FGV.

Cysne, R. P. (2006). On the positive correlation between income and patience. Revista Brasileira de Economia, 60(1):21-32.

McCall, J. J. (1970). Economics of information and job search. Quarterly Journal of Economics, 84:113-126.

Mortensen, D. T. \& Pissarides, C. A. (1994). Job creation and job destruction in the theory of unemployment. Review of Economic Studies, 61:397-415.

Pissarides, C. (1974). Risk, job search and income distribution. The Journal of Political Economy, 82(6):1255-1267.

Stokey, N. L., Lucas Jr., R., \& Prescott, E. C. (1989). Recursive Methods in Economic Dynamics. Harvard University Press. 